\title{
Calibration and signal reconstruction in the ATLAS Tile Hadronic calorimeter
}

\author{
R.Febbraro ${ }^{a}$ on behalf of the ATLAS TileCal group

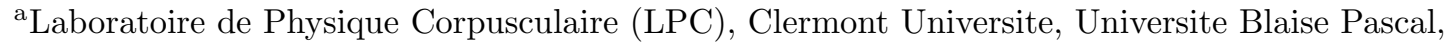 \\ CNRS/IN2P3, Clermont-Ferrand, France
}

TileCal is the central hadronic calorimeter of the ATLAS detector which is one of the four experiments installed at the Large Hadron Collider (LHC) at CERN. In order to calibrate the full read-out path in TileCal different calibration systems are present. The full calibration process relies on three subsystems: the Charge Injection System (CIS), the Laser System, and the Cesium. The signal reconstruction determines the amplitude and the time of the deposited energy. In TileCal the Optimal Filter (OF) algorithm is used for this purpose; in particular the signal is reconstructed in the Read-Out Drivers (ROD) using the Digital Signal Processor (DSP).

\section{The ATLAS Hadronic Calorimeter (TileCal)}

TileCal [1] [2] is the central hadronic calorimeter of the ATLAS [3] detector which is one of the four experiments installed at the Large Hadron Collider (LHC) at CERN. It is a sampling calorimeter made of iron, used as passive material, and plastic scintillators, used as active material. The light produced in the scintillators is sent to photomultipliers by wave-length shifting fibers. The photomultipliers (PMTs) produce an electrical signal which is shaped and digitized by the front-end electronics. The digital signal is sent to the Read-Out Drivers (ROD) boards, through optical fibers, and here it is reconstructed using the Digital Signal Processor (DSP), where the Optimal Filter (OF) algorithm is implemented, and then it is calibrated. The physic signal reconstructed by the OF has an energy precision of $1 \%$.

\section{The Calibration systems}

The response of TileCal is regularly monitored and corrected using a series of calibration systems that focus on each main detector component.

\subsection{The Charge injection system (CIS)}

The CIS [4] generates calibrated amplitude pulses, sent to each electronics channel. It allows to compute the calibration constants for the conversion of the number of ADC counts to $\mathrm{pC}$. Fig. 1 shows the time stability of the electronics for the high gain, both for a typical channel and for the detector wide average. The systematic uncertainty on the individual channels of $\pm 0.7 \%$ is also indicated. The RMS variation for the single channel shown is $0.07 \%$ for the high gain region. The RMS for the detector wide average is $0.03 \%$ for the high gain region.

\subsection{The Laser system}

The Laser System [5] provides calibrated light pulses that are sent to all TileCal PMTs using an optical fiber distribution system. It allowsmeasurement and correction of the gain stability and linearity of the PMT's as a function of time. Fig.2 shows the gain stability over a period of 40 days. The variation is $1.0 \%$ a result that fits with the expected one. The displayed errors of $0.45 \%$ account for both, the statistical uncertainty and the systematic effects. The systematic error coming from the limited reproducibility of the Laser system dominates. 


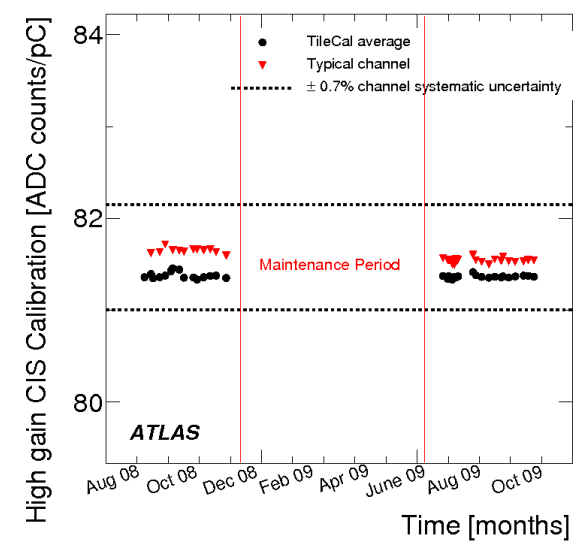

Figure 1. Time stability (1 year) of the average high gain CIS readout calibration constants.

\subsection{The Cesium Calibration}

The Cesium calibration [6] uses a hydraulic system to move a Cesium-137 source through all the TileCal cells. This system is used to set the gain of the TileCal PMTs to correct for non uniformity of the optics elements. Fig. 3 shows the evolution of TileCal response to radioactive Cesium source as function of time for all cells in each section of the calorimeter. The error bars in this plot are the RMS values of the response distribution for all cells in a given partition.

\section{Signal reconstruction}

\subsection{Optimal Filter algorithm}

The Optimal Filtering [7] algorithm estimates the amplitude and the phase of the digitized signal through a weighted sum of digital samples $S_{i}$. In Eqs 1 and 2 the procedure to compute the energy and phase with the OF algorithm is shown.

$$
\begin{aligned}
& A=\sum_{i=1}^{N} a_{i} S_{i} \\
& A \tau=\sum_{i=1}^{N} b_{i} S_{i}
\end{aligned}
$$

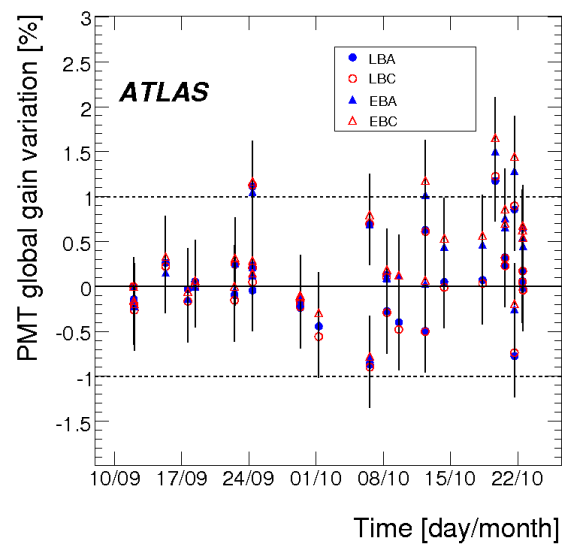

Figure 2. Average gain variation as a function of time.

The amplitude $\mathrm{A}$ is defined as the distance between the peak and the pedestal and the phase $\tau$ is defined as the time between the peaks of the reconstructed pulse and the pulse used for the weights $\left(a_{i}\right.$ and $b_{i}$ in Eqs 1 and 2) computation. Two different kind of OF algorithm are implemented in the DSP:

1. An iterative algorithm that can reconstruct signals which are not synchronous with the trigger.

2. A non-iterative algorithm which requires signals to be synchronous within \pm 1 ns of the trigger (which may be enlarged up to \pm 10 ns by use of a parabolic correction).

\subsection{DSP reconstruction performance}

The performance of the DSP was evaluated using the CIS calibration system. The amplitude of the pulse can be configured to study the performance of the reconstruction algorithm for the whole energy range both in high and low gains. The experimental details of the setup of the ROD and its configuration for use of either online or offline reconstruction of the signals are given in 


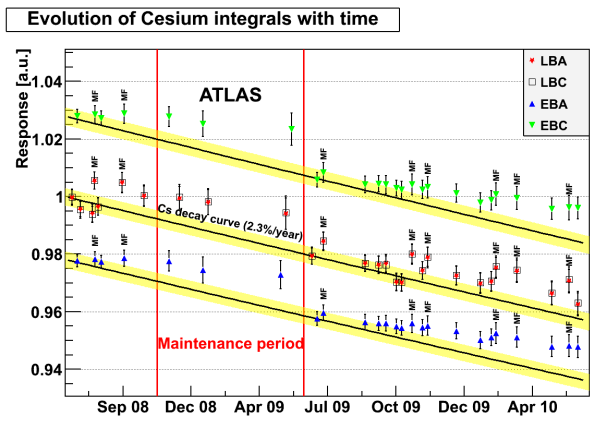

Figure 3. Evolution of TileCal response to radioactive Cesium source as a function of time.

[8]. Fig.4 shows the difference between the energy reconstructed in the DSP and the energy reconstructed offline for the high gain region as a function of the energy reconstructed offline (the high gain range varies from $-1 \mathrm{pC}$ to $15 \mathrm{pC}$ ). It is important to note that the DSP precision is limited by the number of bits used to pack the DSP result. The energy reconstructed in the DSP is packed using 15 bits which implies a maximum precision for the high gain range of about 0.5 $10^{-3} \mathrm{pC}$, in agreement with expectation. Concerning the phase reconstruction the DSP precision is also limited by the number of bits available to pack the result. In this case, the range of phases varies from -64 ns to 64 ns. Because of the fact that TileCal data format uses 10 bits to pack the phase result the precision of the DSP for the phase is $0.0625 \mathrm{~ns}$. Also this result fit with the expected value.

\section{Conclusion}

The calibration systems of the ATLAS hadronic calorimeter show results that fit with the expected ones. In particular the electronic stability measured with the CIS is well within the limit of $\pm 0.7 \%$ of systematic uncertainty. The stability of the gain measured with Laser system is within $1 \%$ and the Cesium calibration shows that optical modules elements work properly. It is important

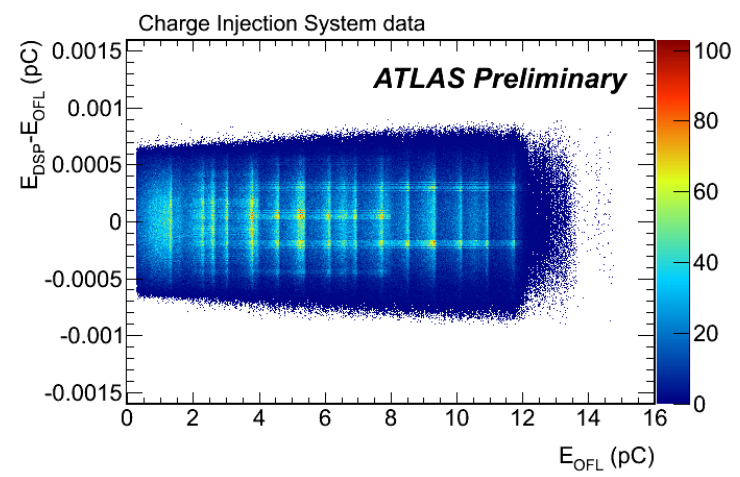

Figure 4. Absolute difference between the amplitude reconstructed online (DSP) and offline as a function of amplitude for CIS data in high gain.

to note that the $\mathrm{OF}$ algorithm which results in a maximum precision of $0.5 \quad 10^{-3} \mathrm{pC}$ for the energy and of $0.0625 \mathrm{~ns}$ for the phase fit with what is expected.

\section{REFERENCES}

1. ATLAS/Tile Calorimeter Collaboration, Technical Design Report, CERN, LHCC/9642, 1996.

2. The ATLAS Collaboration, Readiness of the ATLAS Tile Calorimeter for LHC collisions, submitted for publication to EPJC, 2010.

3. The ATLAS Collaboration, Expected Performance of the ATLAS Experiment at CERN LArge Hadron Collider, JINST 3 (2008) S08003.

4. K. Anderson, et al., Nucl. Instr. and Meth. A 551 (2005) 469.

5. S. Viret, Nucl. Instr. and Meth. A 617 (2010) 120-122.

6. E.A. Starchenko et al., ATL- TILECAL-2002003, CERN 2020.

7. E. Fullana et al., IEEE Trans. Sci, vol.53, no.4, pp.2139-2143, 2006.

8. A. Valero, ATL-TILECAL-PROC-2009-004, CERN 2009. 